\title{
Three-dimensional analysis of surface acoustic resonator for ultra-high sensitive ethanol disclosure as non-invasive biosensor
}

\begin{abstract}
Due to the increasing demand on ethanol gas sensors for several applications such as automation, transportation, or environmental monitoring, the need for more sensitive and reliable sensors with an on-spot response time is required. Indirect sensing, invasive, bulky, time initializing, and low reliability in strip tests are some drawbacks of ethanol sensing measurements at the present. To overcome these leakages, there is focused on modelling and simulation of a proposed ethanol sensor based on surface acoustic wave technique using finite element method.
\end{abstract}

Keywords: surface acoustic resonator, three-dimensional analysis, ethanol detection, non-invasive densor, health monitoring system
Volume I Issue 4 - 2017

\author{
Peyman Jahanshahi,' Qin Wei,' Zhang Jie,' \\ Erfan Zalnezhad ${ }^{2}$ \\ Institute of Intelligent Manufacturing and Information \\ Engineering, Shanghai Jiao Tong University, China \\ ${ }^{2}$ Department of Mechanical Convergence Engineering, Hanyang \\ University, Korea
}

Correspondence: Peyman Jahanshahi, School of Mechanical Engineering, Shanghai Jiao Tong University, 200240 Shanghai,

China, Tel (+86) 13262710 628, Email peyman840@gmail.com

Received: June 27, 2017 | Published: November 21, 2017
Abbreviations: SAW, surface acoustic wave; YLN, yz-lithium niobate; IDT, Interdigital electrodes; SAWGC, gas chromatogram; $\mathrm{TD}$, tin dioxide $\left(\mathrm{SnO}_{2}\right)$; FEM, finite element method; PPM, parts per million.

\section{Introduction}

Surface acoustic wave (SAW) sensors provide desirable characteristics for ethanol detection because of their small size, low fabrication cost, ease of integration and high sensitivity. ${ }^{1-4}$ Unlike surface plasmon resonance (SPR) technique. ${ }^{5-7}$ Which is one of the known optical techniques with high sensitivity in gas detection, SAW can be integrated into a chip-based high throughput arrays due to high working frequency, with affordable expenses. In this study, there is numerically analysed the proposed ethanol sensor based on SAW technology using COMSOL Multiphysics. As presented in Figure 1, the sensor comprises of a YZ-Lithium Niobate $\left(\mathrm{LiNbO}_{3}\right)$ substrate with interdigital electrodes (IDT) patterned on the surface. This sandwichlike structure contains the insulator and sensing medium as well. In this work, aluminium is preferred to be used for IDT material because of its electrical resistance and cost. IDT has two ends including receiver and transmitter end, in which they are geometrically symmetry. The thickness is $200 \mathrm{~nm}$ approximately which makes the IDT to have light mechanical load to the surface and enough lower electrical resistance. The design of the resonator plays an important role in improving mass sensitivity, particularly the sensitive area that supplies a lower detection limit when an optimal configuration is performed in the SAW gas chromatogram (SAW-GC) sensors with the cost reductions as well as higher sensitivity for SAW biological and chemical sensors. Accordingly, a tin dioxide $\left(\mathrm{SnO}_{2}\right)$ layer with a specific thickness is generated on the surface of the sensor because of its high affinity to ethanol molecules. With increasing ethanol absorption on the sensor surface, there is a changing in the density of $\mathrm{SnO}_{2}$ layer as a sensing medium (due to the bound ethanol molecules to $\mathrm{SnO}_{2}$ film) and subsequently produced voltage vibrations in output terminal. Through time-dependent analysis, the frequency response for describing a small variation at output voltage is adequately obtained as shown in Figure 2. There is presented a baseline as a reference along with the spectrum of $20 \mathrm{ppm}$ concentration. With a simple comparison, it can be realized that the intensity change is along the vertical direction at the frequency resonance $(65 \mathrm{MHz})$. It means the amount of material (density of sensing medium) has been changed because of sensing a new concentration of ethanol (with 20ppm). It is obvious the intensity variation of other ethanol concentration can be easily calculated according to the baseline.

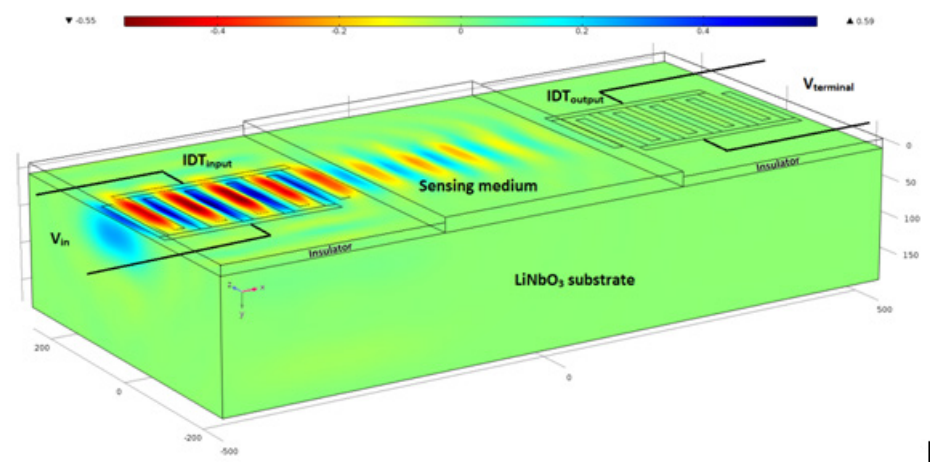

Figure I The three-dimensional structure of a $\mathrm{LiNbO}_{3}$-based SAW device and the process of the exciting acoustic. 


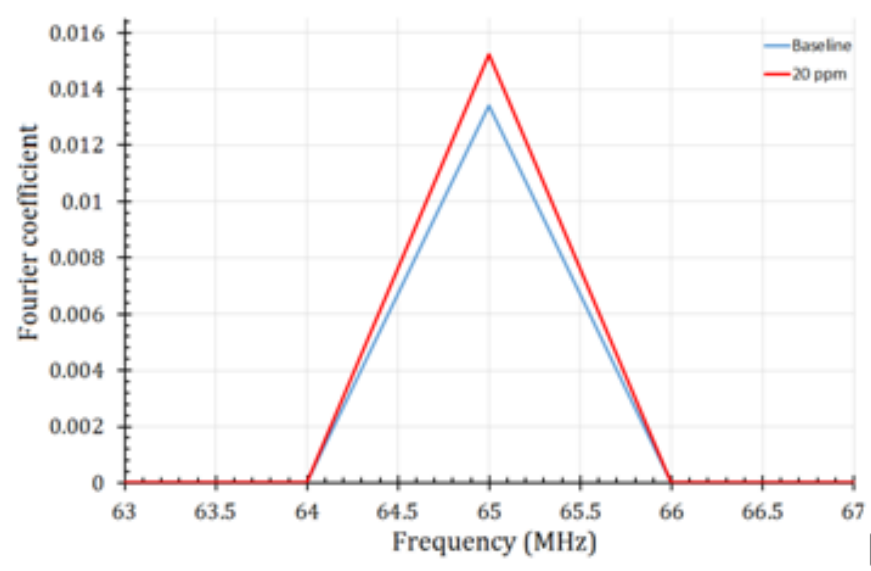

Figure 2 The ID plot of frequency response of the terminal voltage.

\section{Conclusion}

There was employed the SAW configuration for sensing ethanol gas in low concentrations. The proposed sensor could successfully sense a tiny change in physical properties of sensing medium in variety of intended concentrations. The significant contributions of this research work are some unique points which are included ultra-high sensitive ethanol disclosure, ease in integration and mass production, an affordable ethanol sensor, and ability of being as an ethanol warning indicator in specific environments. Since the sensor has an ultra-high sensitivity to the ethanol with fast response, there is expected to utilize this ethanol sensor in special applications as environmental monitoring in medical diagnostics or some other applications like non-invasive controlling alcohol/drug drivers in automobiles. Fabrication of the sensor is particularly highlighted in integration process which will be considered in the close future.

\section{Acknowledgements}

This work has been supported by National Science Foundation of China (No. 51435009).

\section{Conflict of interest}

The authors declare that they have no conflict of interest.

\section{References}

1. Zhang A, Liu W, Jiang Z, et al. Rapid concentration of particle and bioparticle suspension based on surface acoustic wave. Appl Acoust. 2009;70(8):1137-1142.

2. Ding X, Li P, Lin S, et al. Surface acoustic wave microfluidics. Lab Chip. 2013;13(18):3626-3649.

3. Xuan W, He M, Meng N, et al. Fast response and high sensitivity $\mathrm{ZnO}$ glass surface acoustic wave humidity sensors using graphene oxide sensing layer. Sci Rep. 2014;4:7206.

4. Kabir KM, Sabri YM, Matthews GI, et al. Selective detection of elemental mercury vapor using a surface acoustic wave (SAW) sensor. Analyst. 2015;140(6):5508-5517.

5. Jahanshahi P, Parvizi A, Mahamd Adikan FR. Three-dimensional modeling of surface plasmon resonance based biosensor. Germany: OSA, 2013.

6. Jahanshahi P, Mahamd Adikan FR. Sensitivity Enhancement of Graphene-Based Surface Plasmon Resonance Biosensor Using Germanium Nanowires Grating. J Med and Bioeng. 2015;4(2):145-149.

7. Jahanshahi P, Dermosesian E, Mahamd Adikan FR, et al. Numerical and analytical investigation of a SPR structure as Biosensor. Frontiers in Optic. USA; 2014 